\title{
Comprehensive Evaluation of Neuromorphic Computing
}

\author{
Zerksis Mistry $^{1}$, Debjyoti Saha ${ }^{2}$, Omkar Mhapankar $^{3}$, Shashikant Patil $^{4^{*}}$, Suresh Kurumbanshi ${ }^{5}$ \\ ${ }^{1,2,3}$ EXTC DEPT MPSTME Shirpur Campus, Shirpur. \\ ${ }^{4}$ Independent researcher \&amp; Fellow Member IETE, Delhi India \\ ${ }^{5}$ Independent researcher \& amp; IETE, Delhi India
}

*Corresponding Author: Shashikant Patil, Independent researcher \&amp; Fellow Member IETE, Delhi India

\begin{abstract}
Due to technological advancements in the field computing and networking as well, neuromorphic computing has been evolving more and more fast. Advance technologies such as neural and peripheral nerves in human body are growing explosively. If this trend goes on the computing networks congestion will increase and it would be difficult to supply large services to the needy patients. To go on with the flow without any traffic problems neuromorphic computing is the best solution. This paper aims to discuss evaluate address the methods to assess various neuromorphic computing system. Here authors are discussing resistive switching and its properties, gallium doped $\mathrm{ZnO}$ and behaviour of memristive resistors which are playing crucial role in neuromorphic computing. It's an attempt to address the new systems and their role as well as various issues associated with it which are contributing the computing domain.
\end{abstract}

Keywords: neuromorphic computing, neural, memristive resistors, VLSI,

\section{INTRODUCTION}

\subsection{Neuromorphic Computing}

Computation in its many forms is the engine that fuels our modern civilization. Modern computation based on the von Neumann architecture has allowed, until now, the development of continuous improvements, as predicted by Moore's law. However, computation using current architectures and materials will inevitably within the next 10 years reach a limit because of fundamental scientific reasons. The development of novel functional materials and devices incorporated into unique architectures will allow a revolutionary technological leap toward the implementation of a fully "neuromorphic" computer. Computers have become essential to all aspects of Modern life from process controls, engineering, and science to entertainment and communications and are omnipresent all over the globe. Currently, about $5-15 \%$ of the world's energy is spent in some form of data manipulation, transmission, or processing. In the early 1990s, researchers began to investigate the idea of "neuromorphic" computing [15-18]. Nervous system--- inspired analog computing devices were envisioned to be a million times more power efficient than devices being developed at that time. While conventional computational devices had achieved notable feats, they failed in some of the most basic tasks that biological systems have mastered, such as speech and image recognition. Hence the idea that taking cues from biology might lead to fundamental improvements in computational capabilities. Since that time, Researchers have said witnessed unprecedented progress in CMOS technology that has resulted in systems that are significantly more power efficient than imagined. Systems have been mass produced with over 5 billion transistors per die, and feature sizes are now approaching $10 \mathrm{~nm}$. These advances made possible a revolution in parallel computing. Today, parallel computing is commonplace with hundreds of millions of cell phones and personal computers containing multiple processors, and the largest supercomputers having CPU counts in the millions. "Machine learning" software is used to tackle problems with complex and noisy datasets that cannot be solved with conventional "non-learning" algorithms [19,20]. Considerable progress has been made recently in this area using parallel processors. These methods are proving so effective that all major Internet and computing companies now have "deep learning" the branch of machine learning that builds tools based on deep (multilayer) neural networks research opus. Moreover, most major 
research universities have machine learning groups in computer science, mathematics, or statistics [21-24]. Machine learning is such a rapidly growing field that it was recently called the "infrastructure for everything." Neuromorphic computing systems are aimed at addressing these needs. They will have much lower power consumption than conventional processors and they are explicitly designed to support dynamic learning in the context of complex and unstructured data [32-36]. Early signs of this need show up in the Office of Science portfolio with the beginning of instrument erudition created approaches applied to glitches where old-fashioned tactics are derisory. These approaches have been cast-off to investigate the facts formed from weather mockups, in exploration of multifarious configurations not understandable to individuals. They have been cast-off to distinguish structures in bulky measure cosmology records, where the facts capacities are too big for humanoid examination $[25,26]$. They have been used to predict maintenance needs for accelerator magnets so they can be replaced before they fail to search for rare events in high energy physics experiments and to predict plasma instabilities that might develop in fusion reactors. These novel approaches are also being used in biological research from searching for novel features in genomes to predicting which microbes are likely to be in a given environment at a given time. Machine learning methods are also gaining traction in designing materials and predicting faults in computer systems, especially in the so called "materials genome" initiative. Nearly every major research area in the DOE mission was affected by machine learning in the last decade. Today these applications run on existing parallel computers; however, as problems ale and dataset sizes increase, there will be huge opportunities for deep learning on neuromorphic hardware to make a serious impact in science and technology [27-31]. Neuromorphic computing may even play a role in replacing existing numerical methods where lower power functional approximations are used and could directly augment planned Exascale architectures $[37,38,39$, and 40]. Important questions for the future are which areas of science are most likely to be impacted by neuromorphic computing and what are the requirements for those deep neural networks [41-42]. Although this roundtable did not focus on an application driven agenda, it is increasingly important to identify these areas and to further understand how neuromorphic hardware might address them. Non-volatile memory technologies have shown that chalcogenide can change the materials based on germanium-antimony-telluride. Their high speed reads, scalability, less power consumption and multi-level capability. Many studies showing that GSTs-PCMs methods are improving the performance within the new applications. Germanium-antimony-telluride has been proven to be the best material in both optical as well as electrical fields, GST is continuously evolving and is considered to be under development. GST based devices have optimized and achieve an impressive progress in fabrication.

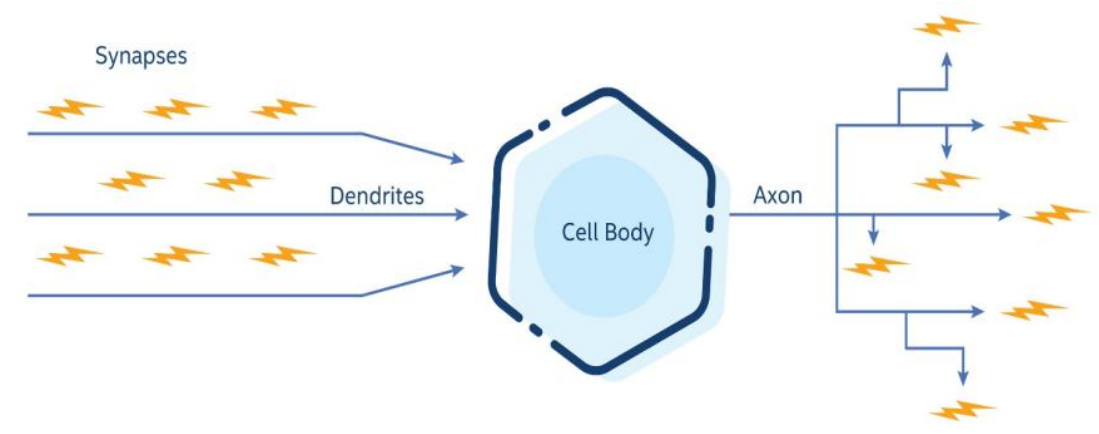

Fig1. Structure of system [48]

Source: https://systemdesign.intel.com/understanding-neuromorphic-computing/

\section{RELATED WORK}

\subsection{Demonstration of Information Transfer in Brain}

Magnetization becomes the most energy efficient and central problem in neuromorphic computing. In last 2 years, many methods have been presented to overcome magnetic switching including spin torque, magneto-electric and microwave-assisted mechanisms. With the help of spin torque mechanism, the nanoscale, ferromagnet increases the rate of thermally-activated switching [3]. Experimentally assisting the chaos and stochasticity in energy, the magnetic Nano-systems paves towards new and efficient spin torque memory. Threshold switching devices are advantageous to number of applications such as memories and neuromorphic circuits. The non-linear specifications are assumed to be occurring without any external input or stimulus which is still a debate trending topics. 
To demonstrate such problems, the scientists have used Scanning Joule expansion Microscopy which generally explains electrical conductivity, negative differential resistance system, etc. the materials like steep dependency on current occurs in nanoscale devices, making friendly to computing.

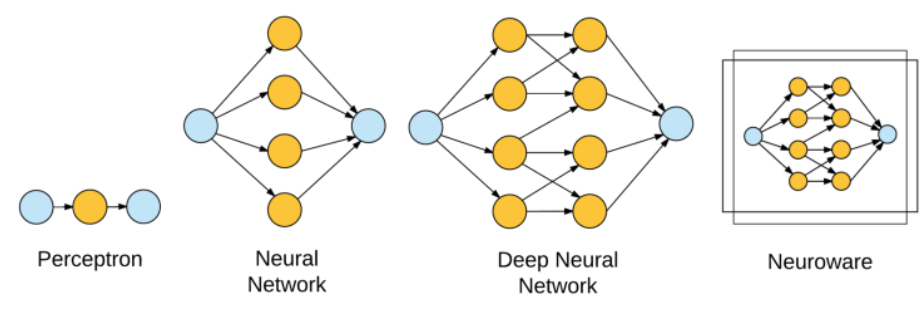

Fig2. decentralized neuromorphic[49]

Source https://blog.chainintel.com/brain-for-machines-ede15dlf24cl

The investigation on random telegraph noise is causing problems in reading of class of memristive devices whose main principles depends on dissolution of conductive elements. The release of electrons from the defects of filament initiates the improper reading of current, which results in low voltages [8]. To overcome this, telegraphic resistances are used in $\mathrm{HfO}_{2}$ memristive devices. The working of telegraphic noise includes demonstration and physical explanation of telegraph noise which intent explains programming operations in identical pulse, neuromorphic computing and stochastic processes. Silver/copper filament is completely depends on the construction of metallic conductive filament (CF). The formation of $\mathrm{CF}$ is so delicate that the lifetime range must be up to few microseconds, or several months, or even years. Conflictive explanations on CF lifetime has been introduced by the disruption of metallic CF.

Triplet based Spike Timing Dependent Plasticity (TSTDP) is an advanced nerve end plasticity rule which can be put into effect by using new Nano-scale technologies. Nano-scale devices which are Nanocrystalline-silicon thin film transistors (TFT) and memristors can be linked into 3 dimensions using low temperature processing [1]. Some of the literatures put forward the new hybrid TFT memristive circuit that is coordinated with TSTDP. The suggested circuit consists of nanoparticle memory-TFTs connected is series as synapse. The results of computer model using spike pairs with several intervals of time and frequencies.

Since 1989, biologically motivated designs have been introduced and in recent days an absorbing field of studies. These brain chips have many technical applications such as pattern recognition, autonomous robotic systems, smart sensors, etc. This Researchers has presented 2 ambipolar nc-Si memory TFTs in series, charged control memristors. To change the synaptic weight through plasticity rules, researchers have focused on PSTDP rule.

A
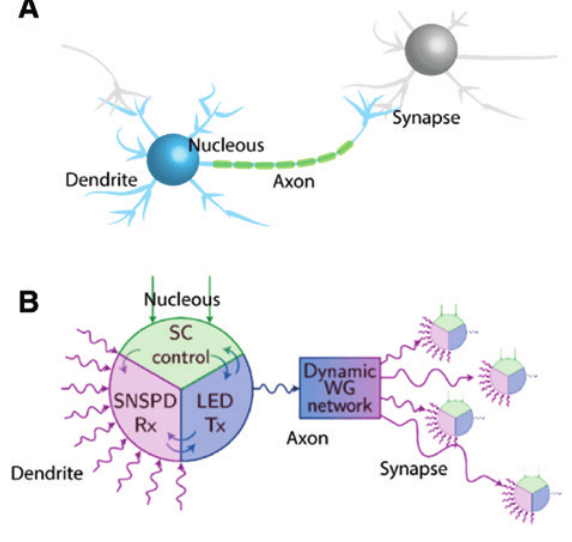

C

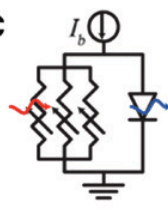

D

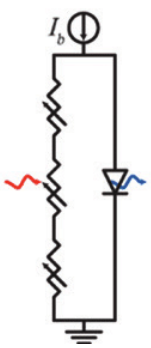

Fig3. Simplified scheme of neuron and hybrid nanophotonic[50]

Source:https://www.researchgate.net/figure/Neuromorphic-computing-with-SNSPDs-A-Simplifiedscheme-of-a-neuron-B-Hybrid_fig13_327778722.

Moving figuring designs from von Neumann to occasion based spiking neural systems (SNNs) reveals new open doors for low-control handling of tangible information in applications, for example, vision or sensorimotor control. Investigating streets toward psychological SNNs requires the structure of 
reduced, low-control what's more, adaptable experimentation stages with the key necessity of web based learning so as to adjust and adapt new highlights in uncontrolled conditions. Nonetheless, implanting internet learning in SNNs is right now blocked by high brought about unpredictability and territory overheads [12]. Many literatures shows that present ODIN, a 0.086-mm2 64kneurotransmitter 256-neuron web based learning computerized spiking neuromorphic processor in 28nm FDSOI CMOS accomplishing a base vitality for each synaptic activity (SOP) of $12.7 \mathrm{pJ}$. It use a proficient usage of the spike-driven synaptic versatility (SDSP) learning rule for high-thickness inserted web based learning with as it were $0.68 \mu \mathrm{m}^{2}$ per 4-bit neural connection. Neurons can be freely designed as a standard defective coordinate and-flame display or as a custom phenomenological show that imitates the 20 Izhikevich practices found in organic spiking neurons. Utilizing a solitary introduction of $6 \mathrm{k} 16 \times 16$ MNIST preparing pictures to a solitary layer fully connected 10-neuron coordinate with on-chip SDSP-based learning, ODIN accomplishes an arrangement precision of $84.5 \%$, while expending just $15 \mathrm{~nJ} /$ deduction at $0.55 \mathrm{~V}$ utilizing rank request coding. ODIN along these lines empowers further advancements toward intellectual neuromorphic gadgets for low-control, versatile and minimal effort handling. The unique and novel parallel registering abilities of the human mind can be imitated with the assistance of neuromorphic figuring approach and this can be accomplished by building up the electronic synaptic gadget. In the present work, Researchers have said incorporated gallium-doped $\mathrm{ZnO}$ slight movies utilizing a financially savvy aqueous technique furthermore, described the dainty movies utilizing field-discharge checking electron microscopy and vitality dispersive $\mathrm{X}$-beam spectroscopy. Moreover, gallium-doped $\mathrm{ZnO}$ memristive gadgets were created utilizing standard technique what's more, electrically portrayed for the neuromorphic application. Specifically, resistive exchanging and synaptic properties of gallium-doped $\mathrm{ZnO}$ slender movies were explored. The bipolar resistive exchanging with a simple memory like conduct was seen in the created memristive gadgets [10]. Few contributions in liteartures shows that, great synaptic properties, perseverance, and maintenance attributes were watched for $0.5 \% \mathrm{Ga}$ doped memristive gadget. They also proposed that the synaptic weight, potentiation-sadness, and symmetric Hebbian learning can be tuned with legitimately designing the $\mathrm{ZnO}$ memristive gadget with proper gallium doping. The nitty gritty investigation of I-V results recommended that resistive exchanging is happened because of Ohmic and Schottky conduction systems.

With effective exchanging of charge is a focal issue in nonvolatile attractive capacity and attractive neuromorphic registering. In previous two decades, a few effective strategies for attractive exchanging were shown including turn torque, magneto-electric, what's more, microwave-helped exchanging instruments. Many researchers claim that low dimensional attractive mayhem prompted by exchanging turn torque can unequivocally build the rate of thermally-actuated attractive exchanging in a nanoscale ferromagnet. Many instrument show a very much articulated limit character in turn torque sufficiency and its proficiency increments with diminishing twist torque recurrence. Some of them quantitatively clarify these exploratory discoveries and uncover the key pretended by lowdimensional attractive confusion close seat equilibria in improvement of the exchanging rate. Few findings on this divulges a critical transaction among tumult and stochasticity in the vitality helped exchanging of attractive nanosystems and makes ready towards improved vitality productivity of turn torque memory and rationale.

Thin films of Pro.6Ca0.4MnO 3 were set up by beat laser testimony with an non identical pair of Silver( $\mathrm{Ag})$ and aluminum( $\mathrm{Al})$ metal terminals so as to study about their resistive switching properties. The gadgets showed stable voltage controlled bipolar changing which ended up being secure and nonvolatile i.e. retaining data even if there is a break in the power supply. The resistive states demonstrate an all-round characterized reliance on the compose voltage, which was utilized to accomplish a few middle states, demonstrating that the gadgets could be used in equipment executions of neuromorphic computing [9]. The switching process was ascribed to the electric-field helped relocation of oxygen opportunities at the Al-terminal interface, bringing about an arrangement and balance of a correcting interfacial AlOx layer. The current-voltage attributes were broke down by methods for the power example portrayal, which implied to a gadget state subordinate exchange of mass restricted PooleFrenkel conduction and interface-constrained Schottky conduction. A more profound comprehension of resistive exchanging or say switching qualities in $\mathrm{Ag} / \mathrm{Pr} 0.6 \mathrm{Ca} 0.4 \mathrm{MnO} 3 / \mathrm{Al}$ will lead towards further advances in manganite-based neuromorphic circuits.

To abridge, RS gadgets were produced from the perovskite $\operatorname{Pr} 0.6 \mathrm{Ca} 0.4 \mathrm{MnO} 3$ with a deviated pair of $\mathrm{Ag}$ and $\mathrm{Al}$ metal interfaces. The devices showing stable bipolar switching with different transitional 
resistive states. The switching was appeared to be non-unstable and repeatable. The conduction display examination demonstrates an exchange of mass constrained Poole-Frenkel conduction and interface restricted Schottky conduction relying upon the gadget state, implying to an adjustment of the AlOx hindrance thickness due to field-helped oxygen opportunity relocation. The opposition states appear a very much characterized reliance on compose voltage which takes into consideration a determination of obstruction esteems between two constraining qualities when a appropriate programming arrangement is utilized. The staggered switching makes the respective device a contender for neuromorphic memory applications, albeit further work is required in streamlining the gadgets furthermore, exploiting their conduction components so as to better copy the uncommon functionalities of organic frameworks.

Many literatures citing inalienable biorealistic synaptic pliancy works in the $\mathrm{Pt} / \mathrm{n}-\mathrm{ZnO} / \mathrm{SiO} 2-\mathrm{x} / \mathrm{Pt}$ heterostructures, where the $\mathrm{n}-\mathrm{ZnO}$ semiconductor is geometrically cone-molded in the span of a couple of nanometers. The synaptic capacities were accomplished inside a two-terminal electroforming and low-control amending diode-like resistive exchanging devices. The imperative rate-subordinate synaptic capacities, for example, the nonlinear transient conduction conduct, shortand long haul versatility, pairing of pulse faciliation, spike-rate-subordinate pliancy and sliding edge impact, were researched in a solitary gadget. These qualities firmly impersonate the memory and learning elements of those in biosynapses, where recurrence subordinate indistinguishable spiking tasks are generally occurring, and we imitate these attributes in the "Learning-Forgetting-Relearning" synaptic conduct. The exchanging elements in the cone-formed $\mathrm{n}-\mathrm{ZnO}$ semiconductor are connected with the vehicle instrument along the grain limits of the charged particle species, in particular, oxygen opportunities and charged oxygen [7]. The dissemination and age/recombination of these imperfections have explicit time sizes of self-rot by goodness of the hilter kilter profile of the $\mathrm{n}-\mathrm{ZnO}$ cone deserts. At long last, the basic biorealistic synaptic versatility capacities were found for the viewpoints of dynamic/versatile electronic neurotransmitter executions in equipment based neuromorphic registering.

The biorealistic rate-subordinate synaptic versatility, which intently imitates the memory/learning pliancy in natural frameworks, has been shown in the redressing diode-like $\mathrm{Pt} / \mathrm{n}-\mathrm{ZnO} / \mathrm{SiO}_{2} \mathrm{x} / \mathrm{Pt}$ synaptic heterostructures. The imperative synaptic pliancy practices, for example, STP and LTP memory maintenance attributes, PPF marvel, SRDP rule, and $\theta \mathrm{m}$ impact, all the while exist in the gadget. The STP and LTP maintenance showing to be very much fitted with exponential and double exponential rot capacities, looking like "human memory" learning and overlooking qualities inside well defined time scales. The PPF wonder alongside the SRDP learning rule were found to comparatively pursue the versatility conduct of that in biorealistic neurotransmitter, because of the two basic highlights of the gadget, as pursues: first, the slow conductance change in the SET/RESET activities; what's more, second, the inner ionic elements, i.e., dispersion what's more, collection of the oxygen opportunities and charged oxygen, which empower simple transient conductance conduct changes by means of bringing down the Schottky diode hindrance stature. The $\theta \mathrm{m}$ impact is investigated to demonstrate the significance of the biorealistic synaptic weight dynamic soundness when various spiking trains with either HFS or LFS are connected to the gadget; be that as it may, the sliding limit impact progressively administers the following synaptic weight update as indicated by the historical backdrop of connected spiking trains. At long last, by using the above synaptic marvels, we effectively imitate the biorealistic "Getting-Forgetting- Relearning" synaptic conduct. Later on, these discoveries could empower versatile and dynamic equipment based neuromorphic frameworks working in a style like that of our cerebrum working.

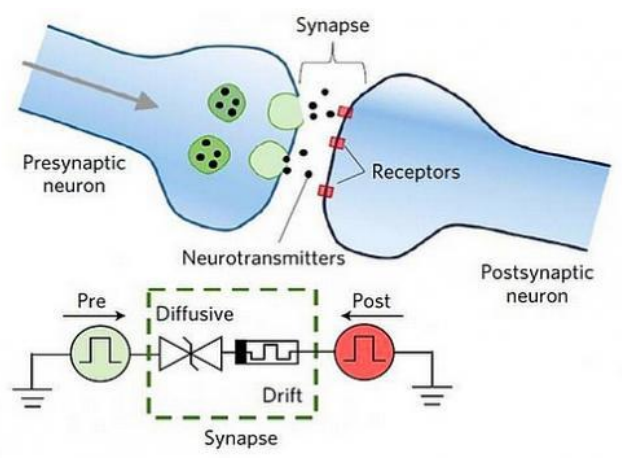

Fig4. Communication mechanism [51] 
Source:https://www.eenewseurope.com/news/memristors-synaptic-emulators-hold-promise-neuromorphiccomputing

The customary Von Neumann engineering based PCs are considered to be deficient in the coming man-made consciousness time because of expanding calculation intricacy and rising force utilization. Neuromorphic processing might be the key job to copy the human cerebrum works and dispose of the Von Neumann bottleneck. As an essential unit in the sensory system, a neural connection is in charge of transmitting data between neurons. Resistive arbitrary get to memory (RRAM) can emulate the synaptic capacities due to its tunable resistive exchanging conduct [13]. Here, a counterfeit neurotransmitter dependent on arrangement handled poly vinyl pyrrolidone (PVPy) - Au nanoparticle (NP) half breed is created, different synaptic capacities including Paired Pulse Facilitation (PPF), Post Tetanic Potentiation (PTP), Short Term Plasticity (STP) i.e. change from transient versatility to Long Term Plasticity (LTP) i.e. long haul versatility and getting the hang of overlooking relearning process are copied, making the polymer- metal NPs half breed framework significant competitors for the plan of novel counterfeit neural structures.

An electronic neurotransmitter (e-neural connection) in view of memristive exchanging is a promising electronic component that copies a natural neurotransmitter to acknowledge neuromorphic processing. Be that as it may, the complex resistive exchanging process it depends on hampers the reproducibility of its performance. In brief, they exhibited a ultrathin e-neural connection that had a high return $(>95 \%)$, a superior consistency, and low power utilization and that worked essentially based on controllable Fowler-Nordheim burrowing. The high gadget yield, device to-device consistency, and cycle-to-cycle consistency are credited to the capacity to precisely control the thickness of the $\mathrm{Al}_{2} \mathrm{O}_{3}$ film when it is stored utilizing ALD and to the uniform appropriation of GQDs on the outside of the $\mathrm{Al}_{2} \mathrm{O}_{3}$ film.

\section{DISCUSSIONS AND FINDINGS}

With the ever-increasing demand for low power electronics, neuromorphic computing has garnered huge interest in recent times. Implementing neuromorphic computing in hardware will be a severe boost for applications involving complex processes such as image processing and pattern recognition [4]. Artificial neurons form a critical part in neuromorphic circuits, and have been realized with complex complementary metal-oxide-semiconductor (CMOS) circuitry in the past. In summary, many researchers have presented a novel 2D material-based TSM comprising v-MoS2 layers grown on a monolayer graphene template. The device mimics the characteristics of a biological neuron via volatile resistive threshold switching behavior. The developed IF artificial neuron exhibits the key behaviors of a biological neuron which include an all or nothing spiking, a threshold driven spiking of the action potential, a post-firing refractory period of a neuron and a strength modulated frequency response.

Many literatures are claiming synthesized gallium-doped $\mathrm{ZnO}$ thin films using a cost-effective hydrothermal method and characterized the thin films using field-emission scanning electron microscopy and energy-dispersive X-ray spectroscopy. Few of the contributions by various researchers suggested that the synaptic weight, potentiation-depression, and symmetric Hebbian learning can be tuned with properly engineering the $\mathrm{ZnO}$ memristive device with appropriate gallium doping. In few cases, good synaptic properties, endurance, and retention characteristics were observed for $0.5 \% \mathrm{Ga}$ doped memristive device. In particular, resistive switching and synaptic properties of gallium-doped $\mathrm{ZnO}$ thin films were investigated. Furthermore, gallium-doped $\mathrm{ZnO}$ memristive devices were developed using standard procedure and electrically characterized for the neuromorphic application. The unique parallel computing capabilities of the human brain can be mimicked with the help of neuromorphic computing approach and this can be achieved by developing the electronic synaptic device. The bipolar resistive switching with an analog memory like behavior was observed in the developed memristive devices. Few contributors effectively orchestrated and described the $\mathrm{ZnO}$ and $\mathrm{Ga}$-doped $\mathrm{ZnO}$ memristive gadgets for the neuromorphic application [2]. The outcomes of findings recommended that the Ga-doping adjusts the morphological and basic properties of $\mathrm{ZnO}$ slender movies. The morphological outcomes recommended that the as-arranged $\mathrm{ZnO}$ dainty film indicates sporadic micro rod structures though, uniform development of 1-D micro rods was watched for 0.5 at.\% Interestingly, change in the morphology from micro rod to micron needle was watched, as the Ga doping increments in the example. Double particulate composites of cobalt ferrite scattered 
in a completely thick PZT grid are created by quite fast sintering of precisely enacted powders. By high-vitality processing of the powders a bi-modular grain measure dissemination, with octahedral nano-grains and bigger grains developed by numerous parallel twinning, are accomplished in the last microstructure. Completely thick PZT-cobalt ferrite double particulate composites can be created by very quick sintering of precisely actuated powder blends. This procedure is the effective reaction to the difficulties of the distinctive shrinkage rates, warm development befuddle, and interface in homogeneities shown by particulate composites [14]. The processing venture of Nb-doped PZTcobalt ferrite powder blend appears to build the relative thickness regardless of the $\mathrm{PbO}$ misfortune because of the expanded molecule reactivity and higher Nb-doped PZT/cobalt ferrite interfaces thickness.

\section{CONCLUSION}

The efficiency and importance of neuromorphic computing is addressed here. The various aspects computing domain and its viability and deployment are mainly focused from the perspective of increasing efficiency and reliability. Main facet of neuromorphic computing is accepting the morphology of distinct neurons, circuits, systems, solicitations, and inclusive designs forms anticipated computations, upsets how statistics is embodied, effects sturdiness to impairment, integrates erudition and improvement, acclimatizes to native alteration (elasticity), and expedites innovative transformation.

\section{REFERENCES}

[1] Soraya Aghnout, Gholamreza Karimi, "Modelling triplet spike timing dependent plasticity using a hybrid TFT-memristor neuromorphic synapse". Integration, the VLSI Journal 64 (2019) 184-191.

[2] Pengfei Guo, Andrew M. Sarangan, Imad Agha, "A Review of Germanium- Antimony-Telluride Phase Change Materials for Non-Volatile Memories and Optical Modulators". Applied Sciences 2019.

[3] Wei Wang, Ming Wang, Elia Ambrosi, Alessandro Bricalli, Mario Laudato, Zhong Sun, Xiaodong Chen \& Daniele Ielmini, "Surface diffusion-limited lifetime of silver and copper nanofilaments in resistive switching devices". Nature Communications 2019.

[4] Eric Arturo Montoya, Salvatore Perna, Yu-Jin Chen, Jordan A. Katine, Massimiliano d'Aquino, Claudio Serpico \& Ilya N. Krivorotov, "Magnetization reversal driven by low dimensional chaos in a nanoscale ferromagnet". Nature Communications 2019.

[5] Jonathan M. Goodwill, Georg Ramer, Dasheng Li1, Brian D. Hoskins, Georges Pavlidis, Jabez J. McClelland, Andrea Centrone, James A. Bain \& Marek Skowronski, "Spontaneous current constriction in threshold switching devices". Nature Communications 2019.

[6] Stefano Brivio, Jacopo Frascaroli, Erika Covi \& Sabina Spiga, "Stimulated Ionic Telegraph Noise in Filamentary Memristive Devices". Scientific Reports.

[7] Shi-Rui Zhang, Li Zhou, Jing-Yu Mao, Yi Ren, Jia-Qin Yang, Guang-Hu Yang, Xin Zhu, Su-Ting Han,* Vellaisamy A. L. Roy,* and Ye Zhou*, "Artificial Synapse Emulated by Charge Trapping-Based Resistive Switching Device". Advanced materials Technology 2019.

[8] Shilpa S. Morea,Pratiksha A. Patila, Kalyani D. Kadama, Harshada S. Patila, Snehal L. Patila, Aishwarya V. Pawara, Sharon S. Kanapallya, Dhanashri V. Desaia, Shraddha M. Bodakea, Rajanish K. Kamatb, Sungjun Kimc,*, Tukaram D. Dongalea, "Resistive switching and synaptic properties modifications in gallium-doped zinc oxide memristive devices". Results in Physics 12 (2019) 1946-1955.

[9] Charlotte Frenkel, Jean-Didier Legat, "A 0.086- $\mathrm{mm}^{2}$ 12.7-pJ/SOP 64k-Synapse 256-Neuron OnlineLearning Digital Spiking Neuromorphic Processor in 28-nm CMOS". IEEE TRANSACTIONS ON BIOMEDICAL CIRCUITS AND SYSTEMS, VOL. 13, NO. 1, FEBRUARY 2019

[10] V. L€ahteenlahti, A. Schulman, H. Huhtinen, P. Paturi, "Transport properties of resistive switching in $\mathrm{Ag} / \mathrm{Pr} 0.6 \mathrm{Ca} 0.4 \mathrm{MnO} 3 / \mathrm{Al}$ thin film structures". Journal of Alloys and Compounds 786 (2019) 84e90.

[11] Kshitij Bhardwaj, Member, IEEE, and Steven M. Nowick, Fellow, IEEE, "A Continuous-Time Replication Strategy for Efficient Multicast in Asynchronous NoCs". IEEE TRANSACTIONS ON VERY LARGE SCALE INTEGRATION (VLSI) SYSTEMS, VOL. 27, NO. 2, FEBRUARY 2019.

[12] Andrey Sergeevich Sokolov, Yu-Rim Jeon, Sohyeon Kim1, Boncheol Ku and Changhwan Choi, "Biorealistic synaptic characteristics in the cone-shaped ZnO memristive device". NPG Asia Materials.

[13] Zhongwei Xu, Fushan Li, Chaoxing Wu, Fumin Ma, Yueting Zheng, Kaiyu Yang, Wei Chen, Hailong Hu, Tailiang Guo and Tae Whan Kim, "Ultrathin electronic synapse having high temporal/spatial uniformity and an Al2O3/graphene quantum dots/A12O3 sandwich structure for neuromorphic computing". NPG Asia Materials. 
[14] Hirokjyoti Kalita, Adithi Krishnaprasad, Nitin Choudhary, Sonali Das, Durjoy Dev, Yi Ding, Laurene Tetard, Hee-Suk Chung, Yeonwoong Jung \& Tania Roy, "Artificial Neuron using Vertical MoS2/Graphene Threshold Switching Memristors". Scientific Reports.

[15] Monroe, D. (2014). "Neuromorphic computing gets ready for the (really) big time". Communications of the ACM. 57 (6): 13-15. doi:10.1145/2601069.

[16] Zhao, W. S.; Agnus, G.; Derycke, V.; Filoramo, A.; Bourgoin, J. -P.; Gamrat, C. (2010). "Nanotube devices based crossbar architecture: Toward neuromorphic computing". Nanotechnology. 21 (17): 175202. Bibcode:2010Nanot.21q5202Z. doi:10.1088/0957-4484/21/17/175202. PMID 20368686.

[17] Mead, Carver (1990). "Neuromorphic electronic systems". Proceedings of the IEEE. 78 (10): 1629-1636. doi:10.1109/5.5835

[18] Maan, A. K.; Jayadevi, D. A.; James, A. P. (January 1, 2016). "A Survey of Memristive Threshold Logic Circuits". IEEE Transactions on Neural Networks and Learning Systems. PP (99): 1734-1746. arXiv:1604.07121. doi:10.1109/TNNLS.2016.2547842. ISSN 2162-237X. PMID 27164608

[19] "A Survey of Spintronic Architectures for Processing-in-Memory and Neural Networks", JSA, 2018

[20] Zhou, You; Ramanathan, S. (August 1, 2015). "Mott Memory and Neuromorphic Devices". Proceedings of the IEEE. 103 (8): 1289-1310. doi:10.1109/JPROC.2015.2431914. ISSN 0018-9219

[21] Boddhu, S. K.; Gallagher, J. C. (2012). "Qualitative Functional Decomposition Analysis of Evolved Neuromorphic Flight Controllers". Applied Computational Intelligence and Soft Computing. 2012: 1-21. doi: $10.1155 / 2012 / 705483$

[22] Farquhar, Ethan; Hasler, Paul. (May 2006). A field programmable neural array. IEEE International Symposium on Circuits and Systems. pp. 4114-4117. doi:10.1109/ISCAS.2006.1693534. ISBN 978-07803-9389-9.

[23] Poon, Chi-Sang; Zhou, Kuan (2011). "Neuromorphic silicon neurons and large-scale neural networks: challenges and opportunities". Frontiers in Neuroscience. 5: 108. doi:10.3389/fnins.2011.00108. PMC 3181466. PMID 21991244

[24] Sharad, Mrigank; Augustine, Charles; Panagopoulos, Georgios; Roy, Kaushik (2012). "Proposal For Neuromorphic Hardware Using Spin Devices". arXiv:1206.3227

[25] Pickett, M. D.; Medeiros-Ribeiro, G.; Williams, R. S. (2012). "A scalable neuristor built with Mott memristors". Nature Materials. 12 (2): 114-7. Bibcode:2013NatMa..12..114P. doi:10.1038/nmat3510. PMID 23241533

[26] Boahen, Kwabena (April 24, 2014). "Neurogrid: A Mixed-Analog-Digital Multichip System for LargeScale Neural Simulations". Proceedings of the IEEE. 102 (5): 699-716. doi:10.1109/JPROC.2014.2313565

[27] Waldrop, M. Mitchell (2013). "Neuroelectronics: Smart connections". Nature. 503 (7474): $22-4$. Bibcode:2013Natur.503...22W. doi:10.1038/503022a. PMID 24201264

[28] Benjamin, Ben Varkey; Peiran Gao; McQuinn, Emmett; Choudhary, Swadesh; Chandrasekaran, Anand R.; Bussat, Jean-Marie; Alvarez-Icaza, Rodrigo; Arthur, John V.; Merolla, Paul A.; Boahen, Kwabena (2014). "Neurogrid: A Mixed-Analog-Digital Multichip System for Large-Scale Neural Simulations". Proceedings of the IEEE. 102 (5): 699-716. doi:10.1109/JPROC.2014.2313565

[29] Modha, Dharmendra (August 2014). "A million spiking-neuron integrated circuit with a scalable communication network and interface". Science. 345 (6197): 668-673. Bibcode:2014 Sci...345..668M. doi:10.1126/science.1254642. PMID 25104385

[30] Davies, Mike; et al. (January 16, 2018). "Loihi: A Neuromorphic Manycore Processor with On-Chip Learning". IEEE Micro. 38 (1): 82-99. doi:10.1109/MM.2018.112130359. Retrieved August 5, 2018

[31] Morris, John. "Why Intel built a neuromorphic chip|ZDNet". ZDNet. ZDNet. Retrieved August 17, 2018

[32] Maan, A.K.; James, A.P.; Dimitrijev, S. (2015). "Memristor pattern recogniser: isolated speech word recognition". Electronics Letters. 51 (17): 1370-1372. doi:10.1049/el.2015.1428

[33] Maan, Akshay Kumar; Kumar, Dinesh S.; James, Alex Pappachen (January 1, 2014). "Memristive Threshold Logic Face Recognition". Procedia Computer Science. 5th Annual International Conference on Biologically Inspired Cognitive Architectures, 2014 BICA. 41: 98-103. doi:10.1016/j.procs.2014.11.09

[34] Maan, A.K.; Kumar, D.S.; Sugathan, S.; James, A.P. (October 1, 2015). "Memristive Threshold Logic Circuit Design of Fast Moving Object Detection". IEEE Transactions on Very Large Scale Integration (VLSI) Systems. 23 (10): 2337-2341. arXiv:1410.1267. doi:10.1109/TVLSI.2014.2359801. ISSN 10638210

[35] James, A.P.; Francis, L.R.V.J.; Kumar, D.S. (January 1, 2014). "Resistive Threshold Logic". IEEE Transactions on Very Large Scale Integration (VLSI) Systems. 22 (1): 190-195. arXiv:1308.0090. doi:10.1109/TVLSI.2012.2232946. ISSN 1063-8210 
[36] James, A.P.; Kumar, D.S.; Ajayan, A. (November 1, 2015). "Threshold Logic Computing: MemristiveCMOS Circuits for Fast Fourier Transform and Vedic Multiplication". IEEE Transactions on Very Large Scale Integration (VLSI) systems. 23 (11): 2690-2694. arXiv: 1411. 5255.doi:10. 1109/TVLSI.2014. 2371857. ISSN 1063-8210

[37] Caravelli et al. (2017). "The complex dynamics of memristive circuits: analytical results and universal slow relaxation". Physical Review E. 95 (2): 022140. arXiv:1608.08651. Bibcode:2017PhRvE..95b2140C. doi:10.1103/PhysRevE.95.022140. PMID 28297937

[38] Patil et al."Mobile Devices Based Mechanisms in Telemedicine and Healthcare: A Systematic Approach" International Journal of Innovative Research in Electronics and Communications Volume 5, Issue 4 (DOI: http://dx.doi.org/10.20431/2349-4050.0504003)

[39] Patil et. al. "Comprehensive Study \& Overview of Neural Networks for Classification of Diseases" International Journal of Innovative Research in Electronics and Communications Volume 5, Issue 3, 2018 , PP 19-27 ISSN 2349-4050 (Online) \& ISSN 2349-4042 (Print) DOI: http://dx.doi.org/10.20431/23494050.0503003

[40] Pravin et.al. "Wireless Power Transmission for Charging Mobile Devices Using Microwave" International Journal of Innovative Research in Electronics and Communications Volume 5, Issue 3, 2018, PP 1-6; ISSN 2349-4050 (Online) \& ISSN 2349-4042 (Print)

[41] Patil et. al."Coherent Access and its Methods in Communication Engineering"International Journal of Innovative Research in Electronics and Communications (IJIREC); ISSN 2349 - 4042 (Print) \& ISSN 2349 - 4050 (Online); DOI: http://dx.do i.org/10.20431/2349 - 4050.0304004

[42] www.thelowdownblog.com

[43] www.degruyter.com

[44] www.dl.sciencesocieties.org

[45] Rahimi Azghadi, Mostafa, Said Al-Sarawi, Derek Abbott, and Nicolangelo Iannella. "A

[46] neuromorphic VLSI design for spike timing and rate based synaptic plasticity", Neural Networks, 2013.

[47] onlinelibrary.wiley.com

[48] https://systemdesign.intel.com/understanding-neuromorphic-computing/

[49] https://blog.chainintel.com/brain-for-machines-ede15d1f24c1

[50] https://www.researchgate.net/figure/Neuromorphic-computing-with-SNSPDs-A-Simplified-scheme-of-aneuron-B-Hybrid_fig13_327778722

Citation: Shashikant Patil, et.al, (2019). "Comprehensive Evaluation of Neuromorphic Computing ". International Journal of Innovative Research in Electronics and Communications (IJIREC), 6(2), pp.17-25. DOI: http://dx.doi.org/10.20431/2349-4050.0602003.

Copyright: (c) 2019 Authors. This is an open-access article distributed under the terms of the Creative Commons Attribution License, which permits unrestricted use, distribution, and reproduction in any medium, provided the original author and source are credited. 